\title{
ENRICHED ENVIRONMENT EFFECTS ON REMOTE OBJECT RECOGNITION MEMORY
}

\author{
RICCARDO MELANI, ${ }^{\text {a* }}$ GABRIELE CHELINI, ${ }^{b}$ \\ MARIA CRISTINA CENNII ${ }^{a}$ AND NICOLETTA BERARDI ${ }^{\mathrm{a}, \mathrm{b}}$ \\ a Institute of Neuroscience of the National Research Council of \\ Italy, 56124 Pisa, Italy \\ ${ }^{\mathrm{b}}$ Department of Neuroscience, Psychology, Drug Research and \\ Child Health (Neurofarba), University of Florence, 50135 \\ Florence, Italy
}

\begin{abstract}
Since Ebbinghaus' classical work on oblivion and saving effects, we know that declarative memories may become at first spontaneously irretrievable and only subsequently completely extinguished. Recently, this timedependent path toward memory-trace loss has been shown to correlate with different patterns of brain activation. Environmental enrichment (EE) enhances learning and memory and affects system memory consolidation. However, there is no evidence on whether and how EE could affect the time-dependent path toward oblivion. We used Object Recognition Test (ORT) to assess in adult mice put in EE for 40 days (EE mice) or left in standard condition (SC mice) memory retrieval of the familiar objects 9 and 21 days after learning with or without a brief retraining performed the day before. We found that SC mice show preferential exploration of new object at day 9 only with retraining, while EE mice do it even without. At day 21 SC mice do not show preferential exploration of novel object, irrespective of the retraining, while EE mice are still capable to benefit from retraining, even if they were not able to spontaneously recover the trace. Analysis of c-fos expression $\mathbf{2 0}$ days after learning shows a different pattern of active brain areas in response to the retraining session in EE and SC mice, with SC mice recruiting the same brain network as naïve SC or EE mice following de novo learning. This suggests that EE promotes formation of longer lasting object recognition memory, allowing a longer time window during which saving is present. (c) 2017 IBRO. Published by Elsevier Ltd. All rights reserved.
\end{abstract}

Keywords: enriched environment, object recognition, longterm memory, saving effect, brain activation.

\section{INTRODUCTION}

The life of a memory trace is quite complex, and it crosses many steps from the encoding of information to its

\footnotetext{
${ }^{*}$ Corresponding author.

E-mail address: melani@in.cnr.it (R. Melani).

Abbreviations: ANOVA, analysis of variance; EE, environmental enrichment; ORT, Object Recognition Test; PFA, paraformaldehyde; SS, standard condition.
}

consolidation in a long lasting trace. We know that the process leading to the formation of a long-lasting declarative memory involves different molecular mechanisms and progressive recruitment of brain areas in what is known as system consolidation (Squire and Alvarez, 1995; Frankland and Bontempi, 2005; RomeroGranados et al., 2010; Bonaccorsi et al., 2013). Forgetting, as assessed by absence of spontaneous recall, can be due to at least two reasons: the memory trace is still present, stored in the brain, but inaccessible to recall; or the memory is no longer stored in the brain (Mirman and Britt, 2013). The first to experimentally study oblivion was Herman Ebbinghaus at the end of 1800 . Using lists of non-sense words, he calculated the number of items that he progressively forgot with time, drawing the "oblivion curve". He also developed the concept of "saving", meaning the facilitation to re-learn non-novel items thanks to the past learning, suggesting that, before becoming completely extinguished, a memory trace crosses a stage during which the effects of learning are not completely lost, but the trace is still present, although inaccessible to spontaneous recall (Ebbinghaus, 1885).

Recently, Romero-Granados and coworkers, using Object Recognition Test (ORT), proposed a model in which a declarative memory trace crosses, with time after learning, two stages: a first stage in which it is apparently forgotten, in that it is not spontaneously recoverable, but the effects of learning are not completely lost, in that the long-term memory of the familiar object can be recovered after a brief retraining (Romero-Granados et al., 2010); a second stage in which the trace is unrecoverable even following brief retraining. These two different states of an apparently lost memory, still recoverable following retraining and unrecoverable, correlate with different patterns of brain activation and of plasticity factors expression in specific areas. The model that emerges from these data suggest that following consolidation, a memory trace can be easily recalled within a certain time period, then it is "hidden", seemingly appearing extinguished because not available to free recall, but still available to "assisted" recall and finally becoming no longer retrievable, suggesting total loss of the trace. It is not known whether this time course toward oblivion is predetermined or can be affected by manipulations of the environmental experience, such as that provided by $E E$, which is known to profoundly affect brain plasticity and to enhance learning and memory (Sale et al., 2014).

Many papers have indeed underlined the beneficial effects of EE on memory acquisition and on recovery 
from cognitive deficits, in aged animals or in animal models of neurodegenerative diseases (Van Praag et al., 2000; Duffy et al., 2001; Berardi et al., 2007; Pizzorusso et al., 2007; Bekinschtein et al., 2011; Leger et al., 2012; Sale et al., 2014); however, whether EE could affect the time-dependent path toward oblivion and the "saving" effect is still not known.

The aim of our study is to verify first whether EE allows to form an object recognition memory trace recoverable for a longer time, either under conditions of spontaneous recall or under conditions of assisted recall, distinguishing therefore between different types of oblivion (trace loss and recovery failure) and second to investigate the possible neural substrates for this EE effect. We found that EE promotes formation of longer lasting object recognition memory with respect to SC, slowing down the path toward memory-trace loss and prolonging the time window during which saving is present.

This correlates with a different pattern of active brain areas in response to the retraining session in EE and SC mice.

\section{EXPERIMENTAL PROCEDURES}

\section{Animals and rearing conditions}

A total of 165 adult male and female C57BL/6 mice were used in this study ( $n=82$ males, $n=83$ females). All procedures were approved by the Italian Ministry of Health. Animals were housed in an animal room with a $12 \mathrm{~h} / 12-\mathrm{h}$ light/dark cycle, with food and water available ad libitum, and experiments were performed during the light phase (Berardi et al., 2007). At 2 months of age, animals were assigned to one of the following rearing conditions for 40 days: Environmental Enrichment (EE: $n=84$, males $n=42$, females $n=42$ ) or standard condition (SC: $n=81$, males $n=40$, females $n=41$ ). SC rearing consisted of $26 \times 18 \times 18-\mathrm{cm}$ cages housing $3-5$ animals; EE rearing condition was achieved using large cages $(44 \times 62 \times 28 \mathrm{~cm})$ housing 6-10 animals, containing several food hoppers, one running wheel for voluntary physical exercise, and differently shaped objects (tunnels, toys, shelters, stairs) that were repositioned twice a week and completely substituted with others once a week (Berardi et al., 2007).

Experiments on EE mice begun after 40 days in EE; after the beginning of experiments, no more novel stimuli were inserted in the cages, to avoid interferences with learned objects. The position of objects inside the cages was however changed twice a week to maintain environmental stimulation.

\section{Apparatus}

We run the ORT in a Y-apparatus (Bartko et al., 2010; Leger et al., 2012) with high, homogenous white walls constructed from Perspex to prevent the mouse from looking out into the room, thereby maximizing attention to the stimuli. One arm was used as the start arm, and had a sliding door to allow access to the arena; the other two arms were used to display the objects. All walls were
$30 \mathrm{~cm}$ high; the start arm was $26 \mathrm{~cm}$ long with the sliding door placed at $13 \mathrm{~cm}$ from the arm end. The lateral arms were $18 \mathrm{~cm}$ long and all arms were $10 \mathrm{~cm}$ wide. The apparatus was placed in a silent room within a box with white walls and ceiling; a video camera was mounted above the apparatus and all trials were recorded with the Ethovision software (Noldus 9.0).

\section{Experimental design and behavioral procedures}

The protocol for behavioral tests is outlined in Fig. 1. On the first day (Day 0) mice were habituated to the $Y$ shape arena for $20 \mathrm{~min}$. The learning session (Sample) was performed $24 \mathrm{~h}$ later (Day 1) allowing the mice to explore for $15 \mathrm{~min}$ two identical objects, each placed at the end of the short arms. Exploration time was taken when mice approached the objects with muzzle and paws. The experimenter measuring exploration time was blind to rearing condition and treatment. The test phase was performed the day after the learning session (Day 2) for all animals, except the naïve group described later, to be sure that learning occurred, and then either following 9-day or 21-day interval (Day 9/Day 21 ), depending on the experimental condition, changing one of the two familiar objects (those explored during the sample phase) with a novel one and the other familiar object with an identical one, and allowing the mice to explore them for $5 \mathrm{~min}$.

A total of 42 EE and 42 SC animals performed the test phase at day 9 or 21 (groups 9 days EE, $n=21,10$ males, 11 females; 9 days SC, $n=23,11$ males, 12 females; 21 days EE, $n=21$, 10 males, 11 females; 21 days SC, $n=19,10$ males, 9 females). Some animals performed the test at day 9 or 21 following a brief retraining session at day 8 or 20 (9 days EE-RET, $n=10,5$ males and 5 females; 9 days SC-RET $n=12,6$ males and 6 females; 21 days EE-RET $n=10,5$ males and 5 females; 21 days SC-RET $n=12,6$ males and 6 females) while other animals performed the test without a preceding retraining session ( 9 days EE-NO RET $n=11,5$ males and 6 females; 9 days SC-NO RET $n=11,5$ males and 6 females; 21 days EE-NO RET $n=11,6$ males and 5 females; 21 days SC-NO RET $n=7,4$ males and 3 females). The retraining session consisted in a brief (3 min) exposure to the familiar objects.

To test for a saving effect, the time length of the brief retraining session should not able to give rise per se to a new long lasting memory. We controlled for this subjecting a separate group of animals, 27 EE and 24 $\mathrm{SC}$, to the habituation phase on Day 0 , to a learning phase of $3 \mathrm{~min}$ (EE $n=13,6$ males and 7 females; SC, $n=11,5$ males and 6 females) or $15 \min$ (EE $n=14$, 7 males and 7 females; SC, $n=13,6$ males and 7 females) at Day 1 and to the test phase at Day 2 (see protocol in Fig. 1).

Arena and objects were cleaned up between trials to stop the build-up of olfactory cues. Objects were simple 3D objects derived from everyday living, and their dimensions were $10-20-\mathrm{cm}$ height and $6-8-\mathrm{cm}$ width. To avoid possible spontaneous preferences for one of the objects, the choice of the new and old object and 


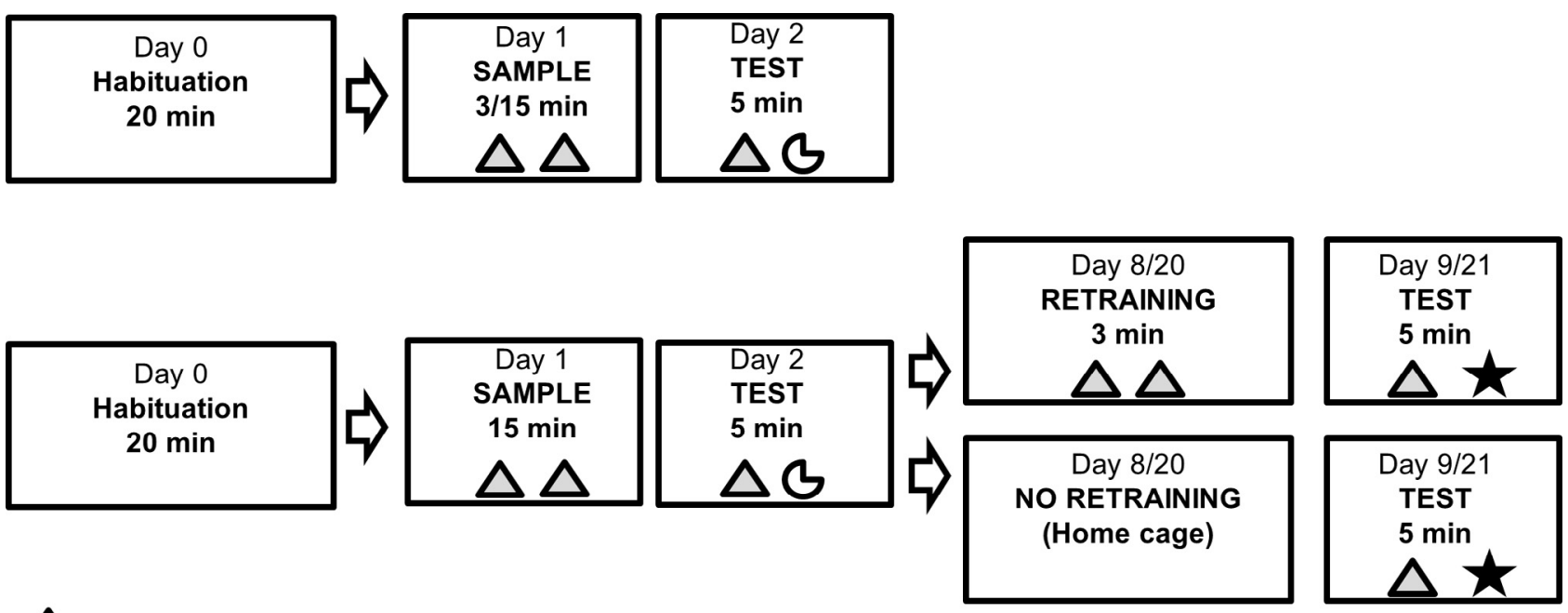

\section{$\triangle$ Sample (familiar) object \\ $\checkmark \quad$ Novel object for $24 \mathrm{~h}$ test \\ Novel object for $9 / 21$ days test}

Fig. 1. Schematic representation of the behavioral test protocol. Top: protocol for assessing the ability of a 3- or 15-min learning phase in giving rise to a long lasting object recognition memory. Bottom: protocol for assessing remote object recognition memory retrieval with or without retraining.
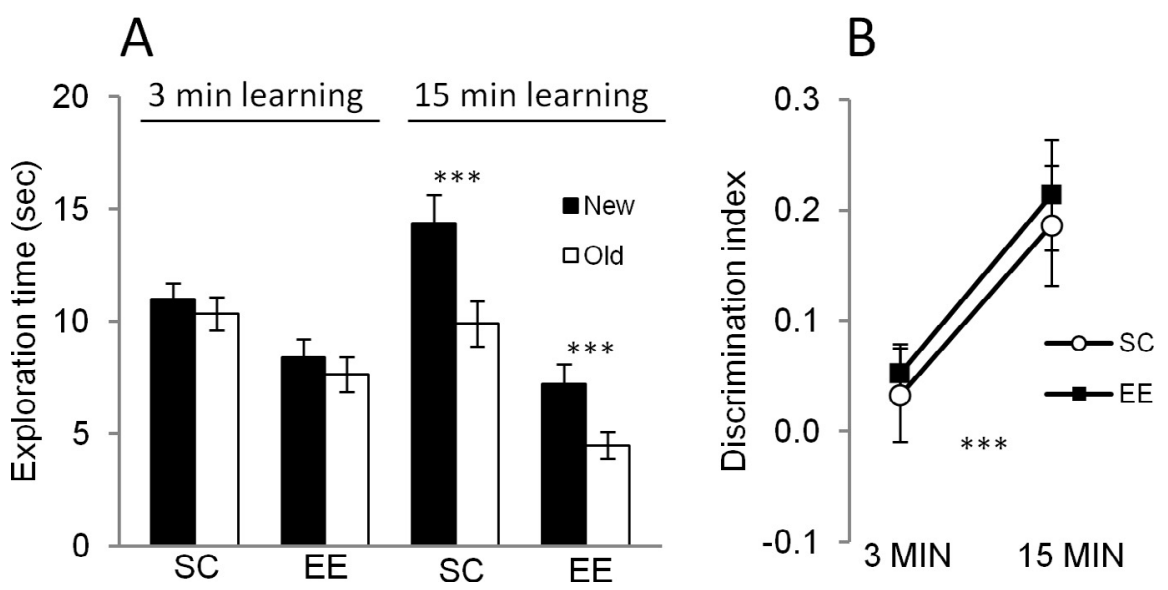

Fig. 2. A 3-min sample phase is not sufficient to allow formation of a long-term memory, while a 15-min session is. (A) Exploration time of novel (New) and familiar (Old) objects did not differ when learning session was 3 min for both groups, while they did when learning session was $15 \mathrm{~min}\left({ }^{* * *}\right)$. (B) Analysis of the discrimination indexes reveals only a main effect of learning duration $\left({ }^{* * *}\right)$, indicating a similar phenotype for SC and EE mice. ${ }^{* * *}$ denotes $p<0.001$. Data are reported as mean \pm s.e.m.

the position of the new one were randomized among animals. Mice exploring the two objects for less than $8 \mathrm{~s}$ during the sample phase were excluded from testing.

The discrimination index was calculated as follows: (TNew - TOld)/(TNew + TOld). TNew and TOld were the time spent exploring the new and the familiar object, respectively.

\section{Immunohistochemistry}

Mice were anesthetized with an overdose of chloral hydrate and then perfused via intracardial infusion with $0.1 \mathrm{M}$ PBS and then $4 \%$ paraformaldehyde (PFA, dissolved in $0.1 \mathrm{M}$ phosphate buffer, $\mathrm{pH} 7.4) 90 \mathrm{~min}$ after the completion of behavioral testing. Brains were removed, fixed overnight in PFA, and then transferred to $30 \%$ sucrose solution and stored at $4{ }^{\circ} \mathrm{C}$. Coronal sections were cut at $40-\mu \mathrm{m}$ thickness on a freezing microtome (Sliding Leica microtome SM2010R, Leica Microsystems, Wetzlar, Germany), and free-floating sections were prepared for immunohistochemistry. After a blocking step in 10\% NGS and $0.5 \%$ Triton $\mathrm{X}-100$ in PBS, sections were incubated in a solution containing $1 \%$ NGS, $0.3 \%$ Triton $X-$ 100 , and anti-c-fos primary rabbit polyclonal antibody (1:3000 rabbit anti c-fos polyclonal antibody, Calbiochem, USA) for $36 \mathrm{~h}$ at $4{ }^{\circ} \mathrm{C}$. Subsequently, sections were transferred in a solution containing $1 \%$ NGS, $0.1 \%$ Triton $X-100$ and 1:200 anti-rabbit biotinylated secondary antibody (Vector Labs, Burlingame, CA, USA) in PBS. This was followed by incubation in ABC kit (Vector Labs, Burlingame, CA, USA) and final detection with DAB reaction kit (Vector Labs, Burlingame, CA, USA). Sections were finally mounted on gelatinized slides, dehydrated and sealed with DPX mounting medium (VWR International, UK).

\section{Analysis of c-fos-positive cells}

Counting of c-fos-positive cells in different brain areas was performed using a CCD camera (MBF Bioscience, 
Germany) mounted on a Zeiss Axioskop (Zeiss, Germany) microscope and the Stereo-Investigator software (MBF Bioscience). Brain structures were anatomically defined according to a mouse brain atlas (Paxinos and Franklin, 1997), and the regions of interest selected for measurement of c-fos-positive nuclei were (numbers indicate the distance in millimeters of the sections from bregma): infralimbic cortex (IL, $+1.54 \mathrm{~mm}$ ); prelimbic cortex (PRL, $+1.54 \mathrm{~mm}$ ); dentate gyrus (DG, $-1.94 \mathrm{~mm}$ ); CA1 field of dorsal hippocampus (CA1, $-1.94 \mathrm{~mm}$ ); CA3 field of dorsal hippocampus (CA3, $-1.94 \mathrm{~mm}$ ); perirhinal cortex ( $\mathrm{PRH},-4.04 \mathrm{~mm})$; primary auditory cortex (AU1, $-3.16 \mathrm{~mm}$ ); primary visual cortex $(\mathrm{V} 1,-3.8 \mathrm{~mm})$; primary motor cortex $(\mathrm{M} 1,+1.54 \mathrm{~mm})$. The number of c-fos-positive cells was counted at $20 \times$ magnification, in $10-40$ fields $(50 \times 50 \mu \mathrm{m}$ or $100 \times 100 \mu \mathrm{m})$ per section according to the size of brain structure, and their density calculated (cells $\left./ \mathrm{mm}^{2}\right)$, using at least five sections for each structure. For each immunostaining experiment, all animals of experimental groups to be compared were processed together. The analysis of the immunostaining involved an initial step of microscope observation of all stained sections from the different groups to establish settings for microscope light intensity and contrast in the Neurolucida. These initial optimized settings were then kept constant to maintain the same exposure through the single images of each section. In addition, to exclude the contribution of background staining and to control for differences in c-fos staining from section to section and from animal to animal we counted only c-fos immunopositive profiles identified as a round dark spot of $\sim 10 \mu \mathrm{m}$ well evident from the background (see Figs. 5 and 6 and Bonaccorsi et al.,
2013). C-fos-positive cells were analyzed with the experimenter blinded to rearing condition and treatment.

Separate groups of EE-RET $(n=6,3$ males and 3 females) and SC-RET ( $n=6,3$ males and 3 females) mice were used for the immunostaining. During the retraining at Day 20, object exploration time of SC and EE mice did not differ, ( $t$-test, SC $n=6$, EE $n=6$, $t_{10}=0.52, p>0.05$ ), making unlikely that differences in object exploration between groups could affect c-fos expression. To control for the extra exposure to the objects and extra handling in the RET groups we introduced two other experimental groups, namely EEPseudo-RET ( $n=3,2$ males and 1 female) and SCPseudo-RET mice $(n=3,2$ males and 1 female). These animals have been put for the same time as EEor SC-RET mice in the arena at Day 20 , but the arena did not contain any object.

For every brain region, we normalized the density of cfos-positive cells measured in each EE-RET and SC-RET mouse to the mean density of c-fos-positive cells in EEPseudo-RET and SC-Pseudo-RET mice, respectively. In this way we can control for differences in c-fos expression simply due to extra handling, exposure to the $\mathrm{Y}$ maze, motor effects in the RET group and isolate the effect of object exploration on c-fos activation.

To assess whether the differences between c-fos expression following the brief 3 min retraining at day 20 in EE and SC mice were due to differences in the state of the memory trace or were present also in naïve mice, we compared c-fos expression in SC and $\mathrm{EE}$ naïve mice either following exploration for $3 \mathrm{~min}$ of the $\mathrm{Y}$-maze containing two equal objects, (SC-naïve-Arena with $\mathrm{Obj}$ mice, $n=3,2$ males and 1 female; EE-naïve Arena with Obj mice, $n=3,1$ male and 2 females) or following exploration for $3 \mathrm{~min}$ of the empty arena (SC-naïve-Empty Arena mice, $n=3,1$ male and 2 females; EE-naïve-Empty Arena mice, $n=3$, 2 males and 1 female). For every brain region, we normalized the density of c-fos-positive cells measured in each EE-Arena with Obj and SC-Arena with Obj mouse to the mean density of c-fos-positive cells in EE-Empty Arena and SCEmpty Arena mice, respectively.

\section{Statistics}

All data are reported as mean \pm s.e. $\mathrm{m}$. We have used SigmaPlot 12.0 for statistical analysis. Normality test (Shapiro-Wilk) and equal variance test were run and satisfied for every statistical test performed. Statistical analysis for familiar and new object exploration time within groups was performed by paired $t$-test. For discrimination indexes and c-fos expression, comparisons between experimental groups were performed by applying a two-way analysis of
Fig. 3. Performance in the ORT 9 days (Day 9) after sample phase with or without a 3-min retraining at Day 8. (A) Exploration time of novel (New) and familiar (Old) objects in SC and EE mice 9 days after learning without any retraining (NO RET condition) or following retraining at day 8 (RET condition). Without retraining, SC mice do not show preferential exploration of New object, while EE mice do $\left({ }^{* * *}\right)$, indicating memory loss for the former at Day 9. Following retraining at day 8 , SC mice explore significantly more the new object $\left(^{* * *}\right)$, indicating recovery of memory trace; EE animals also show preferential exploration of the New object $\left(^{* *}\right)$. (B) Discrimination indexes analysis reveals a significant interaction between rearing condition (SC vs EE) and retraining condition $\left(^{* *}\right)$, with an effect of the retraining presence in the SC group (@) but not in the EE group There is a significant effect of rearing condition within NO RET (\#), and no effect within RET. SC mice can recover an apparently lost memory trace upon a 3-min retraining at day 9 , while EE animals show longer memory retention. The symbols * @\# denote $p<0.05$, ** denotes $p<0.01$ ${ }^{* * *}$ denotes $p<0.001$. Data are reported as mean \pm s.e.m. 
variance (ANOVA) with all pairwise multiple comparison procedures using the suggested post hoc test (HolmSidak test). The number of c-fos-positive cells was also compared to the respective control groups (100\%) with a one-sample $t$-test (Leger et al., 2012). Differences were considered significant when $p<0.05$.

\section{RESULTS}

\section{Choice of the retraining duration}

To test for a saving effect, the time length of the brief retraining session should not able to give rise per se to a new long lasting memory. We controlled for this subjecting a group of animals to a learning phase of 3 or $15 \mathrm{~min}$ at Day 1 and then to the test phase at Day 2 (See protocol in Fig. 1).

As we can see from Fig. 2A, B, we found that 3-min learning in the sample phase originated a long term memory trace neither in SC nor in EE mice; indeed, in the test phase performed $24 \mathrm{~h}$ after the learning phase, neither group did show a preferential exploration of the novel object with respect to the familiar one (Fig. 2A, paired $t$-test, SC $n=11, t_{10}=0.77, p>0.05$; EE, $n=13, t_{12}=2.02$, paired $t$-test, $p>0.05$ ). On the contrary, when learning time in the sample phase was increased to $15 \mathrm{~min}$, both groups showed good recognition memory $24 \mathrm{~h}$ after learning, exploring significantly more the novel object with respect to the familiar one (Fig. 2A, paired $t$-test, SC $n=13$, $t_{12}=4.24, \quad p=0.001 ; \quad \mathrm{EE}, \quad n=14, \quad t_{13}=4.6$, $p<0.001)$. Accordingly, discrimination indexes $24 \mathrm{~h}$ after learning were close to zero following a 3-min learning both for EE and SC mice, while 15-min learning yielded a good memory performance for both (Fig. 2B) (Two-way ANOVA, factors rearing condition and learning duration, no significant main effect of rearing condition $\left(F_{1,47}=0.27, \quad p>0.05\right)$, no significant interaction between rearing conditions and learning duration $\left(F_{1,47}=0.007, p>0.05\right)$ but only a main effect of learning duration (factor learning duration $\left.F_{1,47}=11.96, p=0.001\right)$. We therefore chose $3 \mathrm{~min}$ as the duration of the retraining session, and $15 \mathrm{~min}$ as the duration of the first learning session in the sample phase.

\section{Effect of environmental condition on 9-day-old memory retrieval with or without retraining}

The protocol for assessing remote object recognition memory retrieval with or without retraining is illustrated in Fig. 1.

As shown in Fig. 3A, when tested 9 days after learning, SC mice did not show a preferential exploration of the novel object in absence of the retraining session (exploration time of the novel vs the familiar object, paired $t$-test, SC-NO RET $n=11$, $\left.t_{10}=1.04, p>0.05\right)$. However, if the test was preceded, the day before, by a 3-min retraining session, SC mice explored the novel object significantly more than the familiar one (exploration time of the novel vs the familiar object, paired $t$-test, SC-RET $n=12$, $\left.t_{11}=5.75, p<0.001\right)$.
EE animals showed intact memory for the familiar object when tested the 9th day after learning, exploring the novel one for a significantly longer time even without retraining (exploration time of the novel vs the familiar object, paired $t$-test, EE-NO RET $n=11, t_{10}=4.65$, $p<0.001)$. Retraining did not lead to additional benefits (exploration time of the novel vs the familiar object, paired $t$-test, EE-RET $n=10, t_{9}=3.93, p<0.01$ ). Accordingly, analysis of the discrimination indexes (Fig. 3B) revealed a significant interaction between rearing condition and retraining presence (Two-way ANOVA, factor rearing condition $F_{1,40}=0.103$, $p>0.05$, factor retraining presence $F_{1,40}=4.4$, $p<0.05$, interaction $F_{1,40}=7.46, p<0.01$ ), with an effect of retraining presence evident in the SC $(p<0.05)$ but not in the EE group $(p>0.05)$. Indeed, rearing condition has a significant effect within NO RET $(p<0.05)$, and no effect within RET condition $(p>0.05)$.

\section{Effect of environmental condition on 21-day-old memory retrieval with or without retraining}

We found that 21 days after learning SC mice were unable to recall the familiar object not only in absence of the retraining session, as at day 9 , but also following it (Fig. 4A), (exploration time of the novel vs the familiar object, paired $t$-test, SC-NO RET $n=7, t_{6}=-1.94$, $p>0.05$; SC-RET $n=12, t_{11}=-0.74, p>0.05$ ).

EE animals, even if unable to spontaneously recall the familiar object memory (exploration time of the novel vs the familiar object, paired $t$-test, EE-NO RET $n=11$, $\left.t_{10}=0.17, p>0.05\right)$, did not show a complete loss of the memory trace in that they showed memory retrieval following the retraining session (exploration time of the novel vs the familiar object, paired $t$-test, EE-RET $\left.n=10, t_{9}=3.54, p<0.01\right)$.

Accordingly, discrimination indexes following retraining resulted significantly different between $S C$ and EE mice (Fig. 4B, Two-way ANOVA, factors rearing conditions and retraining presence; factor rearing condition $F_{1,33}=7.82, \quad p<0.01$, factor retraining presence $\quad F_{1,33}=3.23, \quad p>0.05, \quad$ interaction $\left.F_{1,33}=0.24, \quad p>0.05\right)$. No differences were found between the two groups in the condition without retraining ( $p>0.05$, rearing condition within NO RET), but there was an effect of rearing within the RET condition $(p<0.05)$.

\section{C-fos expression in EE and SC mice following retraining session 20 days after learning}

We analyzed c-fos expression 90 min after the retraining session performed 20 days after learning, which is the time point when the retraining is differently effective in SC and EE mice (Fig. 5). We focused on brain regions known to be involved in recognition memory, such as perirhinal cortex, hippocampus and prefrontal cortex. Since many variables as motor activity, attention, handling and exposure to arena contribute to the levels of c-fos expression (Aggleton et al., 2012), we compared EE-RET $(n=6)$ and SC-RET $(n=6)$ mice with EEPseudo-RET and SC-Pseudo-RET mice $(n=3$ in both 

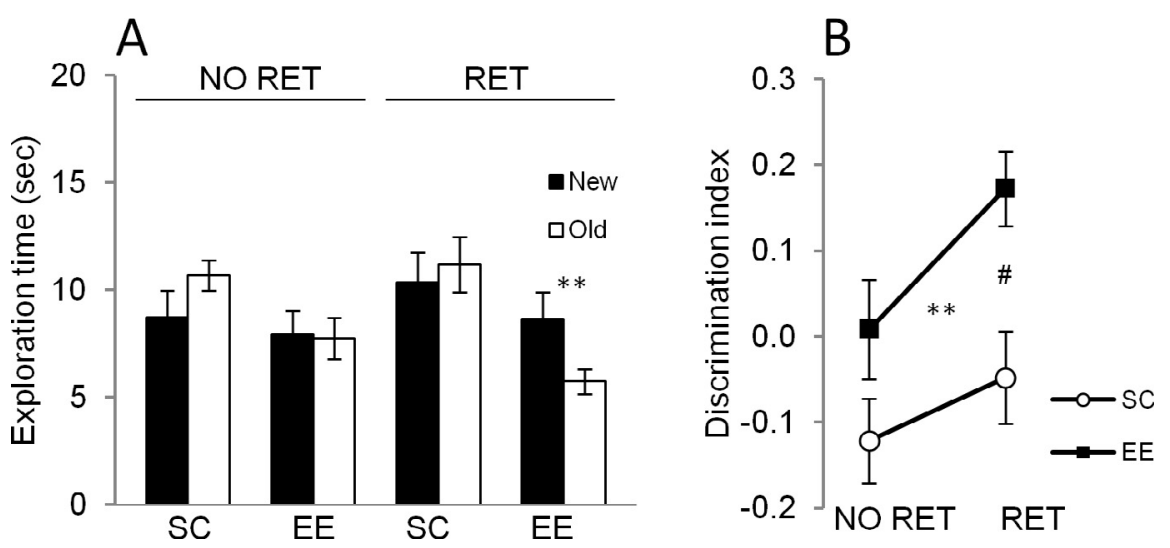

Fig. 4. Performance in the ORT 21 days (Day 21) after sample phase with or without a 3-min retraining at Day 20. (A) Exploration time of novel (New) and familiar (Old) objects in SC and EE mice 21 days after learning without any retraining (NO RET condition) or following retraining at day 20 (RET condition). Without retraining, both SC and EE mice do not show preferential exploration of New object, indicating memory loss at Day 21. However, EE animals still benefit from the retraining, exploring significantly more the new object $\left.{ }^{* *}\right)$ in the RET condition, indicating recovery of memory trace at day 21. SC mice do not benefit from retraining, showing no preferential exploration for the New object even in the RET condition. (B) Discrimination indexes analysis reveals a significant main effect of rearing condition (SC vs EE) $\left(^{* *}\right)$, but no significant effect of retraining presence and no interaction between rearing condition and retraining presence. There is no effect of rearing condition without retraining, but significant effect of rearing upon retraining (\#). 3-min retraining is sufficient to reinstate an apparently lost memory in EE mice at day 21. Symbol conventions for statistical differences as in Fig. 3. Data are reported as mean \pm s.e.m.

groups), i.e. SC and EE animals exposed to the arena for 3 min at day 20 without objects (Leger et al., 2012) (Fig. 5, top inset). No differences in c-fos expression were found between SC-Pseudo-RET and EE-Pseudo-RET mice in any area, suggesting that EE and SC condition per se does not affect c-fos expression in our areas of interest during exposure to the arena (Two-way ANOVA, factors rearing condition and brain area, $\left.F_{8,36}=1.6, p>0.05\right)$.

To compare brain activation in response to retraining between the two groups, SC-RET and EE-RET mice, we calculated, for each region, c-fos expression in animals that underwent the retraining session (SC-RET mice: PRH, CA1, CA3 $n=4$, DG $n=5$, PRL, IL, AU1, V1, M1 $n=3$; EE-RET mice: PRH, PRL, IL, VI, M1, AU1 $n=3$, CA1 $n=4$, DG, CA3 $n=5$ ). For each area, the density of c-fos-positive cells in EERET and SC-RET mice was expressed as a percentage of the mean density of c-fos-positive cells in their respective Pseudo-RET controls. Two-way ANOVA revealed a statistically significant interaction between factors rearing condition and brain area $\left(F_{8,42}=4.03\right.$; $p=0.001)$, with $\mathrm{PRH}, \mathrm{DG}, \mathrm{CA} 1, \mathrm{PRL}, \mathrm{IL}(p<0.001)$ showing higher $\mathrm{c}$-fos expression in SC-RET with respect to EE-RET (Fig. 5).

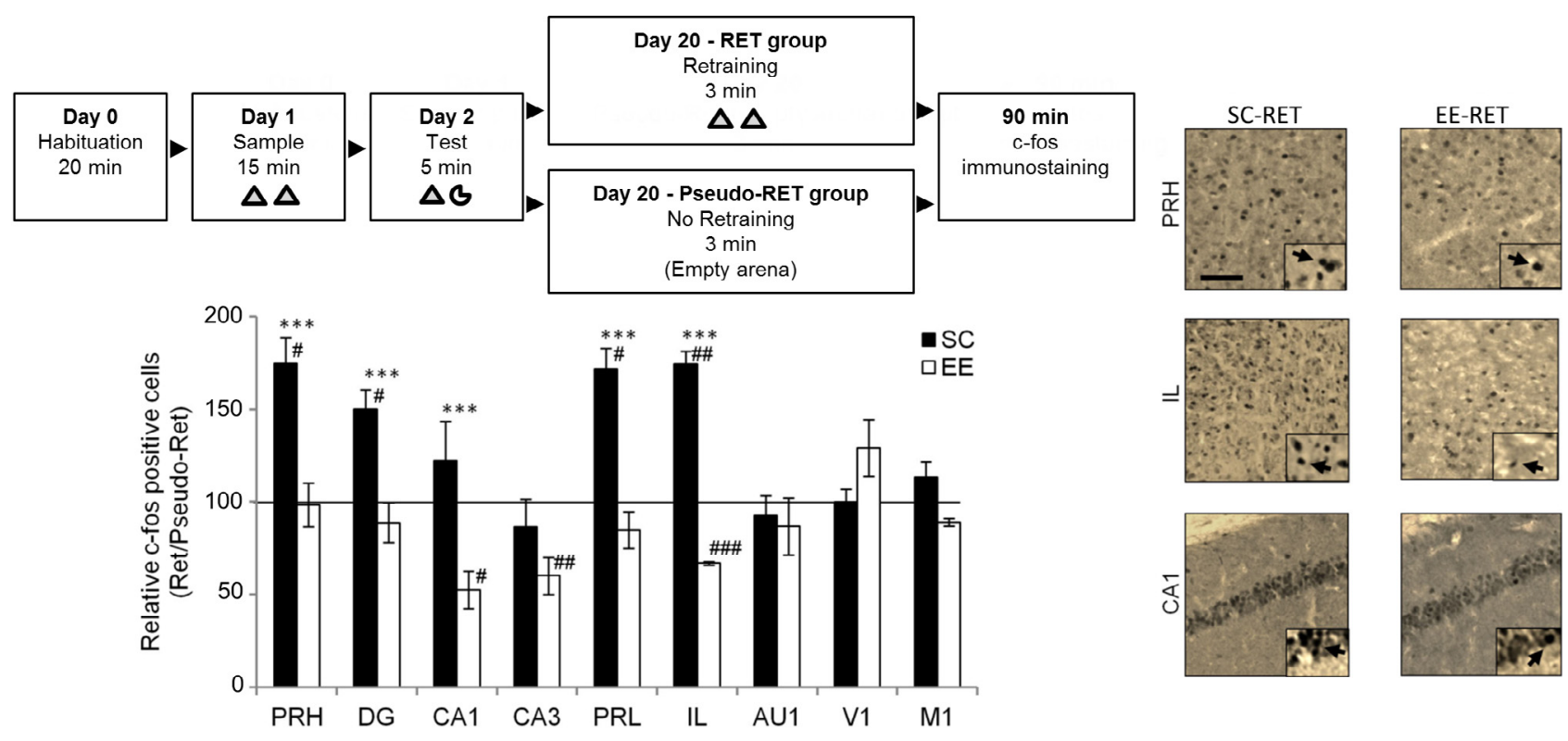

Fig. 5. EE- and SC- RET-mice recruited different brain regions following retraining at day 20. Top inset: protocol for behavioral and immunostaining analysis. The density of c-fos-positive cells in EE-RET and SC-RET mice is expressed as a percentage of the mean density of c-fos-positive cells in their respective Pseudo-RET controls. SC-RET mice showed increased c-fos expression 90 min following retraining with respect to EE-RET mice in $\left.\mathrm{PRH}\left({ }^{* * *}\right), \mathrm{DG}\left(^{* * *}\right), \mathrm{CA} 1{ }^{(* *}\right), \mathrm{PRL}\left({ }^{* * *}\right)$ and IL $\left(^{* * *}\right)$. SC-RET mice displayed an increased c-fos expression with respect to Pseudo-RET-SC mice in PRH (\#), DG (\#), PRL (\#) and IL (\#\#). EE-RET mice showed reduced c-fos activity with respect to Pseudo-RET EE mice in CA1 (\#), CA3 (\#\#) and IL (\#\#\#). Right - Sample images of c-fos immunostaining in PRH, IL and CA1 in SC-RET and EE-RET mice following reminder. 20X magnification, scale bar $=50 \mu \mathrm{m}$. The arrows indicate c-fos-positive cells (magnified). Symbol conventions for statistical differences as in Fig. 3. PRH: Perirhinal cortex; DG: Dentate gyrus; CA1 and CA3, hippocampal CA1 and CA3 regions; PRL: Prelimbic cortex; IL: Infralimbic cortex; AU1: Primary auditory cortex; V1: Primary visual cortex; M1: Primary motor cortex. Data are reported as mean \pm s.e.m. 
Accordingly, c-fos expression upon RET condition resulted significantly higher in SC-RET mice with respect to their SC-Pseudo-RET control group in $\mathrm{PRH}$ (one-sample $t$-test; $\left.t_{3}=5.37 ; p<0.05\right)$, DG $\left(t_{4}=3.3\right.$; $p<0.05)$ PRL $\left(t_{2}=6.33 ; p<0.05\right)$ and IL $\left(t_{2}=10.73\right.$; $p<0.01)$, suggesting that retraining led to neuronal activation in these regions (Fig. 5). EE-RET mice instead showed reduced $\mathrm{C}$-fos expression with respect to their EE-Pseudo-RET controls in CA1 (one-sample $t$ test; $\left.t_{3}=-4.65 ; p<0.05\right)$, CA3 $\left(t_{4}=-5.4 ; p<0.01\right)$ and IL $\left(t_{2}=-39.65 ; p<0.001\right)$, suggesting reduced neuronal activation following retraining in these areas (Fig. 5).

The results of the comparison between EE-PseudoRET and SC-Pseudo-RET mice already suggest that rearing condition does not lead to different c-fos expression in the control condition of the empty arena exploration. To further exclude possible basal differences in c-fos expression simply due to the different housing condition, we investigated c-fos expression in AU1 and V1 sensory cortices and in M1. We did not find differences between c-fos expression in SC-RET and EE-RET mice in these regions (Two-way ANOVA, $\quad F_{8,42}=4.03$; factors rearing condition and area, SC vs EE in AU1, V1 and M1 $p>0.05)$. Even comparing $c$-fos expression of RET-mice with their Pseudo-RET controls, we found no significant differences between RET and Pseudo-RET condition in the SC group or in the EE group for any region (SCRET vs SC-Pseudo-RET, AU1, one-sample $t$-test; $\left.t_{2}=-0.68 ; p>0.05\right)$, V1 $\left(t_{2}=0.006 ; p>0.05\right)$ and M1 ( $t_{2}=1.55 ; p>0.05$ ); (EE-RET vs EE-Pseudo-RET, one-sample $t$-test, $\left.\mathrm{AU} 1 ; \quad t_{2}=-0.87 ; p>0.05\right), \quad \mathrm{V} 1$ $\left(t_{2}=1.9 ; p>0.05\right)$ and $\mathrm{M} 1 \quad\left(t_{2}=-6.83 ; p>0.05\right)$ (Fig. 5).

\section{C-fos expression in naïve EE and SC mice following 3-min object exploration}

To assess whether the differences between c-fos expression following the brief 3-min retraining at day 20 in EE and SC mice were due to differences in the state of the memory trace or might be present also in naïve mice, we compared c-fos expression in naïve mice allowed to explore the empty arena (Empty Arena group, EE $n=3$, SC $n=3$ ) or the arena with a pair of equal objects (Arena with Obj group, EE $n=3$, SC $n=3$ ) for $3 \mathrm{~min}$ (inset Fig. 6). No differences in c-fos expression were found between SC-Empty Arena and EE-Empty Arena mice (Two way ANOVA, factors rearing condition and brain area, $F_{8,36}=1.62, p>0.05$ ).

For each area, the density of c-fos-positive cells in EEArena with Obj and SC-Arena with Obj mice was expressed as a percentage of the mean density of cfos-positive cells in their respective Empty Arena controls. We found no differences between SC and EE animals in activation of brain areas (Two-way ANOVA; factors rearing condition and brain area, no significant effect of rearing condition $F_{1,36}=2.45, p>0.05$; and no interaction between rearing condition and area $\left.F_{8,36}=2.18, p>0.05\right)$. Both for EE and SC groups, higher c-fos expression in Arena with objects mice with respect to Empty Arena mice was found in $\mathrm{PRH}$ (onesample $t$-test; SC: $t_{2}=4.94$; EE: $\left.t_{2}=4.99, p<0.05\right)$, DG (SC: $t_{2}=4.77$; EE $t_{2}=6.47 . p<0.05$ ), PRL (SC: $t_{2}=11.33 ;$ EE: $t_{2}=12.11, p<0.01$ ) and IL (SC: $t_{2}=7.35, p<0.05$; EE: $\left.t_{2}=15.54, p<0.01\right)$, that is the pattern of neuronal activation in SC-RET mice following retraining at Day 20 , suggesting that the latter activation reflects the absence of object memory.

As found for the RET condition, no significant differences between Arena with Obj and Empty Arena groups were found in SC or EE mice in $A U 1, V 1$ and M1: SC, AU1 (one-sample $t$-test; $t_{2}=-0.03 ; p>0.05$ ), V1 $\left(t_{2}=1.53 ; \quad p>0.05\right)$ and $\mathrm{M} 1 \quad\left(t_{2}=-0.14\right.$; $p>0.05) ; \quad$ EE, $\quad\left(\mathrm{AU} 1 ; \quad t_{2}=-0.19 ; \quad p>0.05\right), \quad \mathrm{V} 1$ $\left(t_{2}=-1.34 ; p>0.05\right)$ and $\mathrm{M} 1\left(t_{2}=-0.68 ; p>0.05\right)$.

\section{DISCUSSION}

We found that EE prolongs the "lifespan" of recognition memory, not only in terms of classically assessed long term memory retention (Duffy et al., 2001; Berardi et al., 2007; Leger et al., 2012) but also in terms of latent memory. We employed a behavioral protocol that allows highlighting the presence of a faster re-learning ability of remote memories within a given temporal window from initial learning, reminiscent of the saving effects demonstrated by Ebbinghaus. Indeed the brief retraining is not able by itself to create a de novo long-lasting memory trace in naïve mice but is sufficient to allow formation of a long-lasting memory trace for a previously learned object. Therefore, this protocol allows evaluating the "saving" effect in mice.

Using this protocol, Romero-Granados et al. (2010) proposed a model where the stabilization of an object recognition memory trace occurs by several steps from encoding to reconsolidation mechanisms, recruiting hippocampus and perirhinal cortex both during the first acquisition phase and also following very long-term memory reactivation, performed when the trace is no longer recoverable. This finding highlights how after memory loss, experiencing the forgotten information leads to a recapitulation of the initial consolidation phase, as if that information was new.

We found that EE animals did not differ from SC animals in terms of the ability to remember the familiar object $24 \mathrm{~h}$ after learning, but in the subsequent time course of memory availability for retrieval: their memory remains available for unassisted recovery for a longer time, as shown by the data at Day 9 after learning, when EE mice show preferential exploration of new object without the retraining session. In addition, while memory trace was completely lost for SC mice 21 days after learning, as shown by the lack of effect of the brief retraining, EE animals are still capable of benefiting from retraining, even if they were not able to spontaneously recover the memory trace. This suggests that EE does not affect the initial acquisition process, but promotes formation of longer-lasting object recognition memory, slowing down the path toward memory-trace loss and allowing a longer time window during which saving is present. Indeed, EE mice tested 


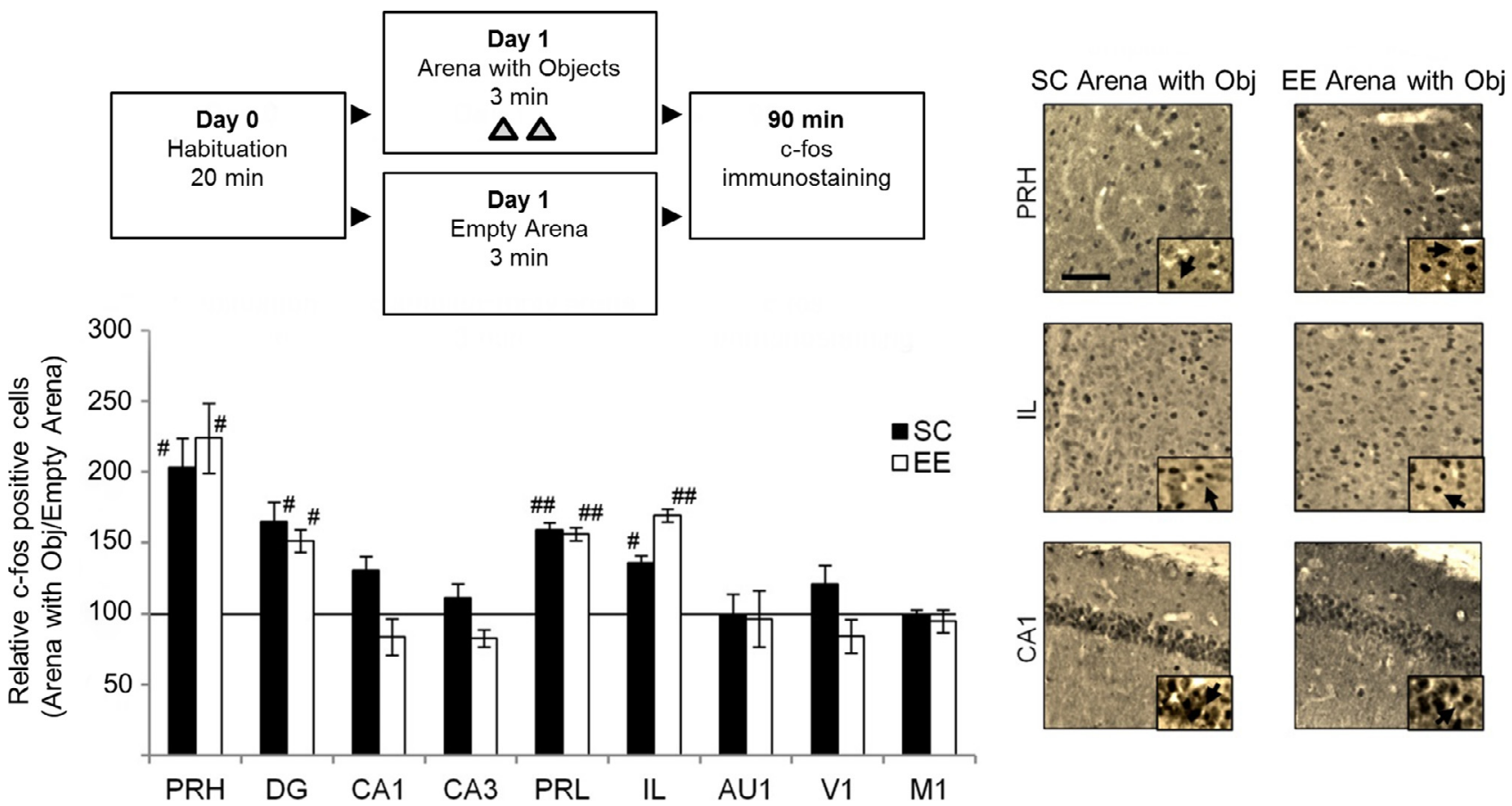

Fig. 6. Naïve EE and SC mice recruited the same brain areas following $3 \mathrm{~min}$ of object exploration. Top inset: protocol for behavioral and immunostaining analysis. The density of c-fos-positive cells in EE-Arena with objects and SC-Arena with objects mice is expressed as a percentage of the mean density of c-fos-positive cells in their respective controls, animals exploring the arena without objects (EE-and SC-Empty Arena groups). Both EE- and SC- Arena with objects mice showed significant c-fos expression with respect to their controls in PRH (SC \#; EE \#), DG (SC \#; EE \#), PRL (SC \#\#; EE \#\#) and IL (SC \#; EE \#\#). No significant difference was found between EE- and SC-Arena with object mice. Right Sample images of c-fos immunostaining in IL, PRH and CA1 in naïve SC and EE mice upon exploration of Arena with objects. $20 \times$ magnification, scale bar $=50 \mu \mathrm{m}$. The arrow indicates c-fos-positive cells (magnified). Symbol conventions for statistical differences as in Fig. 3. PRH: Perirhinal cortex; DG: Dentate gyrus; CA1 and CA3, hippocampal CA1 and CA3 regions; PRL: Prelimbic cortex; IL: Infralimbic cortex; AU1: Primary auditory cortex; V1: Primary visual cortex; M1: Primary motor cortex. Data are reported as mean \pm s.e.m.

21 days after learning behave like SC mice tested 9 days after learning.

It is interesting that employing the classical procedure for object recognition memory testing, SC and EE mice both display forgetting for the remote memory 21 days after learning. However, employing the brief retraining protocol it is possible to reveal a profound difference between these forms of oblivion: SC mice do not show saving, suggesting that the memory trace is by now completely lost, while EE mice show a clear saving, suggesting that a memory trace is still present, albeit latent.

In order to understand by which mechanisms EE could affect memory life span, and how SC and EE mice respond to a re-exposition to lost or unrecoverable information, we assessed c-fos expression upon retraining at a time point where this session exerts a differential role in the two groups, that is 20 days after learning.

We found that perirhinal cortex, prefrontal cortex and hippocampus display differential activation upon a brief retraining session in EE and SC animals. At this time point SC mice recruit a brain network including perirhinal cortex, dentate gyrus and prefrontal cortex. This is the pattern of neuronal activation found in naïve SC mice following exposure for $3 \mathrm{~min}$ to the Arena with objects, that is, following the beginning of a de novo acquisition phase. This pattern of brain activation therefore seems to reflect the absence of an object memory at Day 20 in SC mice.

EE animals, instead, do not show recruitment of perirhinal cortex, dentate gyrus and prefrontal cortex following retraining at day 20: on the contrary, they show reduction in c-fos expression in prefrontal cortex and in CA1 and CA3. This pattern of brain activation also differs from that of naïve EE mice, which activate the same brain areas as SC naïve mice following exposure for $3 \mathrm{~min}$ to the Arena with objects. Since naïve $E E$ and $S C$ mice activate the same brain network during the de novo acquisition phase of object memory, the different pattern of activation found following the 3min retraining at Day 20 suggests that at this time point EE animals experience not completely novel stimuli, even if they were not able to spontaneously recall the familiar object.

We can exclude that the differences in c-fos activation upon retraining at Day 20 between SC and EE animals can stem from basal differences in c-fos activity simply due to the different environmental condition in which mice were reared, since not only naïve SC and EE mice or SC- and EE-Pseudo-RET mice do not differ but control regions (AU1, $\mathrm{V} 1$ and $\mathrm{M} 1$ ) showed no differences between groups following retraining at Day 20. Exploratory activity during the retraining session at Day 20 was also comparable between SC- and EE-RET mice, suggesting that differences in $\mathrm{c}$-fos expression 
between SC- and EE-RET mice could not be attributed to differences in exploratory behavior. We can argue that the different pattern of brain activation in SC and EE mice following retraining at Day 20 is suggestive that in the latter the memory trace is "latent" but not lost, as indicated by the presence of the "saving" effect shown by the successful recall following brief retraining.

The activation of perirhinal cortex, hippocampus and prefrontal cortex in SC mice during de novo learning and the retraining at Day 20 is consistent with the roles of these structures in ORT.

Hippocampus does not seem to be crucial for the familiarization process with the new objects and for object recognition memory, which seem to engage preferentially perirhinal cortex, but it would be employed only if an object-context association is learned (Winters et al., 2004; Norman and Eacott, 2004; Murray et al., 2007; Balderas et al., 2008; Roozendaal et al., 2010), or in object placing recognition (memory for "where", Mendez et al., 2015). There is however evidence that hippocampus would not be completely ineffective in object recognition memory acquisition: for example RomeroGranados et al. (2010) found an increase in hippocampal BDNF after object recognition training, and a role for mTOR signaling in this region has been revealed during consolidation of Object Recognition Memory (Myskiw et al., 2008; Jobim et al., 2012).

An interesting model proposed by Fernández and Tendolkar (2006) suggests that perirhinal cortex acts as a "gatekeeper" for memory processing, regulating hippocampal activity. In this model, information is serially processed via the rhinal cortices, to make efficient familiarity/novelty discriminations before deeper encoding can begin. This information would be then used to regulate encoding and retrieval operations by the hippocampal formation. The model states that when a new item is perceived, a large number of rhinal neurons are required to process this information, leading to the sense of novelty, effective encoding and the effective transfer of information to the hippocampus. In contrast, when a familiar item is recognized, smaller numbers of rhinal neurons are needed to process this item, leading to weaker encoding, reduced information transfer to the hippocampus, and a sense of familiarity.

According to this, familiar stimuli would show reduced activation of the hippocampus; moreover Albasser et al. (2010) show that hippocampus is not engaged upon exploration of familiar stimuli. These findings are consistent with the reduced activation of the hippocampus in EE mice during the retraining session at Day 20, suggesting again that at this time point the memory of the familiar objects is not completely lost.

Enhanced c-fos expression in perirhinal and dentate gyrus for SC mice is probably due to an initial and partial activation of the rhinal-hippocampal circuit, since for SC mice the explored objects are novel again. It may be hypothesized that, since the brief retraining is not sufficient to start a complete consolidation phase, it would fail to recruit the complete hippocampal formation, so that $\mathrm{CA} 3$ and $\mathrm{CA} 1$ are not activated in SC mice.
Perirhinal cortex projects ipsilaterally to the prefrontal cortex (Delatour and Witter, 2002; Hoover and Vertes, 2007), and it has been demonstrated that protein synthesis and N-methyl-D-aspartate (NMDA) function in the ventromedial prefrontal cortex are required for long-term recognition memory and its reconsolidation (Akirav and Maroun, 2006). Moreover, the role of prefrontal cortex has been highlighted also in a recent study by Rossato et al. (2013), in which it was demonstrated that infusions of dopamine $D_{1} / D_{5}$ receptor antagonist in the prefrontal cortex disrupts object recognition memory consolidation. The increased activation of prelimbic and infralimbic cortices observed in the present work in response to the retraining session in SC animals is consistent with these observations. Conversely, EE mice display a global reduction of activity in these regions while exploring objects during the retraining session. This phenomenon is not surprising, considering that the more efficient is the storage, the less activation is required to recall that information. The memory trace for the learned objects is still represented in the brain of EE mice, as revealed by the efficacy of the retraining session, and probably EE mice have no need to recruit a wide c-fos expression during the exploration of non-novel stimuli.

Recently, Epp et al. (2016) found that increased neurogenesis weakens spatial existing memories and, in doing so, facilitates the encoding of new, conflicting information avoiding proactive interference. It is important to note that these authors manipulated hippocampal neurogenesis by providing mice with running wheels after the end of learning an hippocampal dependent task (water maze or associative learning, odor-context), and assessed long-term recall and reversal learning, while in our paradigm, EE was completed before mice began the learning session for the object recognition memory. It is interesting that, as previously reported (Van Praag et al., 2000). Epp et al. (2016) observed an improved acquisition and memory in the spatial memory test when running occurred before training.

In the field of neuropsychology, it is very important to understand if amnesic patients suffer from a loss of memory traces or from a failure in recovery (Rossel and David, 2006; Duarte et al., 2007; Mirman and Britt, 2013). Understanding this difference and the involved physiological mechanisms could help to find new rehabilitation strategies, based on exposition to learned and subsequently lost information.

We can conclude that EE affects not only memory formation and consolidation but also oblivion. EE prolongs the phase of latent memory, which can be uncovered with appropriate experiments, such as the use of a brief re-exposition to familiar items. This means that even when no effects of EE are immediately evident in terms of better memory retention (as for the test at 21 days after learning), it might be important to look deeper into other aspects of the issue, such as, in our case, the "quality" of oblivion. The use of a brief reexposure to the learned material could be a useful strategy to better understand memory deficits in animal studies, and a possible strategy to recover impaired 
performances, allowing us to distinguish between failures in recovery and loss of memory traces.

\section{REFERENCES}

Aggleton JP, Brown MW, Albasser MM (2012) Contrasting brain activity patterns for item recognition memory and associative recognition memory: Insights from immediate-early gene functional imaging. Neuropsychologia 50:3141-3155.

Akirav I, Maroun M (2006) Ventromedial prefrontal cortex is obligatory for consolidation and reconsolidation of object recognition memory. Cereb Cortex 16(12):1759-1765.

Albasser MM1, Poirier GL, Aggleton JP (2010). Qualitatively different modes of perirhinal-hippocampal engagement when rats explore novel vs. familiar objects as revealed by c-Fos imaging. Eur $\mathrm{J}$ Neurosci 31(1):134-47.

Balderas I, Rodriguez-Ortiz CJ, Salgado-Tonda P, Chavez-Hurtado J, McGaugh JL, Bermudez-Rattoni F (2008) The consolidation of object and context recognition memory involve different regions of the temporal lobe. Learn Mem 15(9):618-624.

Bartko SJ, Cowell R, Winters BD, Bussey TJ, Saksida LM (2010) Heightened susceptibility to interference in an animal model of amnesia: impairment in encoding, storage, retrieval - or all three? Neuropsychologia 48:2987-2997.

Bekinschtein P, Omen CA, Saksida LM, Bussey TJ (2011) Effects of environmental enrichment and voluntary exercise on neurogenesis, learning and memory, and pattern separation: BDNF as a critical variable? Sem Cell Dev Biol 22:536-542.

Berardi N, Braschi C, Capsoni S, Cattaneo A, Maffei L (2007) Environmental enrichment delays the onset of memory deficits and reduces neuropathological hallmarks in a mouse model of Alzheimer-like neurodegeneration. J Alzheimer Dis 11:359-370.

Bonaccorsi J, Cintoli S, Mastrogiacomo R, Baldanzi S, Braschi C, Pizzorusso T, Cenni MC, Berardi N (2013) System consolidation of spatial memories in mice: effects of enriched environment. Neural Plast 2013:956312.

Delatour B, Witter MP (2002) Projections from the parahippocampal region to the prefrontal cortex in the rat: evidence of multiple pathways. Eur J Neurosci 15(8):1400-1407.

Duarte LR, Syssau A, Jiménez M, Launay M, Terrier P (2007) Deficit of access or storage: semantic memory processing in Alzheimer disease. Can J Aging 26(3):227-239.

Duffy SN, Craddock KJ, Abel TV, Nguyen P (2001) Environmental enrichment modifies the PKA-dependence of Hippocampal LTP and improves hippocampus-dependent memory. Learn Mem 8 (1):26-34.

Ebbinghaus H (1885). Über das Gedachtnis, Dunker \& Humbolt.

Epp JR, Mera RS, Köhler S, Josselyn SA, Frankland PW (2016) Neurogenesis-mediated forgetting minimizes proactive interference. Nat Commun 7:1-8.

Fernández G, Tendolkar I (2006). Trends Cogn Sci 10(8):358-362.

Frankland PW, Bontempi B (2005) The organization of recent and remote memories. Nat Rev Neurosci 6:119-130.

Hoover WB, Vertes RP (2007) Anatomical analysis of afferent projections to the medial prefrontal cortex in the rat. Brain Struct Funct 212(2):149-179.

Jobim PF, Pedroso TR, Werenicz A, Christoff RR, Maurmann N, Reolon GK, Schröder N, Roesler R (2012) Impairment of object recognition memory by rapamycin inhibition of mTOR in the amygdala or hippocampus around the time of learning or reactivation. Behav Brain Res 228(1):151-158.

Leger M, Quiedeville A, Paizanis E, Natkunarajah S, Freret T, Boulouard M, Schumann-Bard P (2012) Environmental enrichment enhances episodic-like memory in association with a modified neuronal activation profile in adult mice. PLoS One 7: e48043.

Mendez M, Arias N, Uceda S, Arias JL (2015) C-Fos expression correlates with performance on novel object and novel place recognition tests. Brain Res Bull 117:16-23.

Mirman D, Britt AE (2013) What we talk about when we talk about access deficits. Philos Trans R Soc Lond B Biol Sci 369:20120388.

Murray EA, Bussey TJ, Saksida LM (2007) Visual perception and memory: a new view of medial temporal lobe function in primates and rodents. Annu Rev Neurosci 30:99-122.

Myskiw JC, Rossato JI, Bevilaqua LR, Medina JH, Izquierdo I, Cammarota M (2008) On the participation of mTOR in recognition memory. Neurobiol Learn Mem 89(3):338-351.

Norman G, Eacott MJ (2004) Impaired object recognition with increasing levels of feature ambiguity in rats with perirhinal cortex lesions. Behav Brain Res 148(1-2):79-91.

Paxinos G, Franklin K (1997) The mouse brain in stereotaxic coordinates. San Diego, Calif, USA: Academic Press.

Pizzorusso T, Berardi N, Maffei L (2007) A richness that cures. Neuron 54:508-510.

Romero-Granados R, Fontan-Lozano A, Delgado-Garcia JM, Carrion AM (2010) From learning to forgetting: behavioral, circuitry, and molecular properties define the different functional states of the recognition memory trace. Hippocampus 20:584-595.

Roozendaal B, Hernandez A, Cabrera SM, Hagewoud R, Malvaez M, Stefanko DP, Haettig J, Wood MA (2010) Membrane-associated glucocorticoid activity is necessary for modulation of long-term memory via chromatin modification. J Neurosci 30 (14):5037-5046.

Rossato JI, Radiske A, Kohler CA, Gonzalez C, Bevilaqua LR, Medina JH, Cammarota M (2013) Consolidation of object recognition memory requires simultaneous activation of dopamine D1/D5 receptors in the amygdala and medial prefrontal cortex but not in the hippocampus. Neurobiol Learn Mem 106:66-70.

Rossel SL, David AS (2006) Are semantic deficits in schizophrenia due to problems with access or storage? Schizophr Res 82:121-134.

Sale A, Berardi N, Maffei L (2014) Environment and brain plasticity: towards an endogenous pharmacotherapy. Phisiol Rev 94:189-234.

Squire LR, Alvarez P (1995) Retrograde amnesia and memory consolidation: a neurobiological perspective. Curr Opin Neurobiol 5:169-177.

Van Praag H, Kempermann G, Gage FH (2000) Neural consequences of environmental enrichment. Nature Rev Neurosci 1:191-198.

Winters BD, Forwood SE, Cowell RA, Saksida LM, Bussey TJ (2004) Double dissociation between the effects of peri-postrhinal cortex and hippocampal lesions on testsof object recognition and spatial memory: heterogeneity of function within thetemporal lobe. J Neurosci 24:5901-5908. 